\title{
Additivity of Higher Multiplicative Mappings in Rings
}

\author{
RajaaC.Shaheen \\ Department of Mathematics, College of Education ,University of Al-Qadisiyah,Iraq. \\ E.Mail:Rajaa.chaffat@qu.edu.iq
}

Recived : $20 \backslash 4 \backslash 2017$

Revised : $28 \backslash 5 \backslash 2017$

Accepted : 14\6\2017

\begin{abstract}
We study the additivity of higher multiplicative mappings in rings.
\end{abstract}

Mathematics subject classification : .

\section{Introduction}

Let $\mathcal{R}$ and $\mathrm{S}$ be an arbitrary rings with associative property (not necessarily with identity elements). An injective mappings $\sigma$ of $\mathcal{R}$ onto $\mathrm{S}$ such that $(x y)^{\sigma}=x^{\sigma} y^{\sigma}$ for all $\mathrm{x}, \mathrm{y}$ $\in \mathrm{R}$ will be said a "multiplicative isomorphism" of $\mathcal{R}$ onto $\mathrm{S}$. The study of additivity of multiplicative isomorphism has been done by "Rickart[3]" ,"Wang [5]"and also by "Johnson [1]" .In both of these paper some sort of minimality conditions were imposed on the ring R. In [2] the author generalized the main theorem of Rickarts paper [3] and at the same time remove the minimality condition .In [4]Shaheen defined higher multiplicative maps and study its additivity on Triangular rings .In this work, we introduce a study on the additivity of higher multiplicative maps in rings, we should mentioned the reader that our result in this paper is along different lines than those in shaheens results.Now, we shall give Shaheens definition see[ 4] of higher multiplicative maps which is basic in this paper.

\section{Definition 1.1:-[4]}

Let $\mathcal{R}$ and $\mathcal{R}$ be two rings ,a family $\varphi=\left(\varphi_{\mathrm{i}}\right)_{\mathrm{i} \in \mathrm{N}}$ of mappings of a ring $\mathcal{R}$ into $\mathcal{R} . \varphi$ is said to be higher multiplicative if $\forall$ $\mathrm{n} \in \mathrm{N}$.We have

$$
\varphi_{\mathrm{n}}(\mathrm{ab})=\sum_{\mathrm{i}=1}^{\mathrm{n}} \varphi_{\mathrm{i}}(\mathrm{a}) \varphi_{\mathrm{i}}(\mathrm{b}) \text { for all } \mathrm{a}, \mathrm{b} \in \mathcal{R} .
$$

Throughout this section $\varphi_{i}(a) \varphi_{j}(b)=0$ for all $\mathrm{i} \neq j$ and $\varphi_{i}(o)=0$ for all $\mathrm{i}$, suppose that $\mathcal{R}$ be a ring contain $\left\{e_{\alpha}: \alpha \in \Lambda\right\}$ family of idempotent that satisfy:

(i) $\mathrm{x} \mathcal{R}=\{0\}$ implies $\mathrm{x}=0$;

(ii)If $e_{\alpha} \mathcal{R} \mathrm{x}=\{0\} \forall \alpha \in \Lambda$, then $\mathrm{x}=0$ (and hence $\mathcal{R} \mathrm{x}=\{0\} \Rightarrow \mathrm{x}=0$.

(iii) $\forall \alpha \in \Lambda, e_{\alpha} \mathrm{x} e_{\alpha} \mathcal{R}\left(1-e_{\alpha}\right)=\{0\} \Rightarrow e_{\alpha} x e_{\alpha}=0$.

\section{2-Results}

Now, we shall introduce some lemmas

Lemma 2.1 : For all $\mathrm{x}_{\mathrm{ij}} \in R_{i j}$ for all $\mathrm{i}, \mathrm{j}$

$$
\varphi_{\mathrm{k}}\left(\mathrm{x}_{\mathrm{ii}}+\mathrm{x}_{\mathrm{jm}}\right)=\varphi_{\mathrm{k}}\left(\mathrm{x}_{\mathrm{ii}}\right)+\varphi_{\mathrm{k}}\left(\mathrm{x}_{\mathrm{jm}}\right) \quad \mathrm{j} \neq \mathrm{m} .
$$




\section{Proof}

Let $\mathrm{i}=\mathrm{j}=1$ and $\mathrm{m}=2$. Let $\mathrm{z}$ is an element of $\mathcal{R}$ such that

$\varphi_{\mathrm{k}}(z)=\varphi_{\mathrm{k}}\left(\mathrm{x}_{11}\right)+\varphi_{\mathrm{k}}\left(\mathrm{x}_{12}\right)$ for all $\mathrm{k}$.

For arbitrary $\mathrm{a}_{1 \mathrm{j}} \in \mathcal{R}_{1 \mathrm{j}}$, we have

$$
\begin{aligned}
& \varphi_{\mathrm{k}}\left(z \mathrm{a}_{1 \mathrm{j}}\right)=\sum_{i=1}^{k} \varphi_{i}(z) \varphi_{i}\left(a_{1 j}\right) \\
&= \sum_{i=1}^{k}\left(\varphi_{i}\left(x_{11}\right)\right. \\
&\left.+\varphi_{i}\left(x_{12}\right)\right) \varphi_{i}\left(a_{1 j}\right) \\
&= \sum_{i=1}^{k} \varphi_{i}\left(x_{11}\right) \varphi_{i}\left(a_{1 j}\right) \\
&=+\varphi_{i}\left(x_{12}\right) \varphi_{i}\left(a_{1 j}\right) \\
& \sum_{i=1}^{k} \varphi_{i}\left(\left(x_{11}+x_{12}\right)\left(a_{1 j}\right)\right)
\end{aligned}
$$

Since $\varphi_{i}$ is injective, then $z a_{1 j}=\left(x_{11}+\right.$ $\left.x_{12}\right) a_{1 j}$

In the same fashion, for $a_{2 j} \in \mathcal{R}_{2 j}$, we have

$$
\begin{gathered}
\varphi_{\mathrm{k}}\left(\mathrm{z} a_{2 j}\right)=\sum_{\mathrm{i}=1}^{\mathrm{k}} \varphi_{\mathrm{i}}(\mathrm{z}) \varphi_{\mathrm{i}}\left(a_{2 j}\right) \\
=\sum_{\mathrm{i}=1}^{\mathrm{k}}\left(\varphi_{\mathrm{i}}\left(\mathrm{x}_{11}\right)\right. \\
\left.+\varphi_{\mathrm{i}}\left(\mathrm{x}_{12}\right)\right) \varphi_{\mathrm{i}}\left(\mathrm{a}_{2 \mathrm{j}}\right) \\
=\sum_{\mathrm{i}=1}^{\mathrm{k}} \varphi_{\mathrm{i}}\left(\mathrm{x}_{11}\right) \varphi_{\mathrm{i}}\left(\mathrm{a}_{2 \mathrm{j}}\right) \\
+\varphi_{\mathrm{i}}\left(\mathrm{x}_{12}\right) \varphi_{\mathrm{i}}\left(\mathrm{a}_{2 \mathrm{j}}\right) \\
=\varphi_{\mathrm{k}}\left(\left(x_{11}+x_{12}\right) a_{2 j}\right)
\end{gathered}
$$

Since $\varphi_{\mathrm{k}}$ is injective ,then

$$
z a_{2 j}=\left(x_{11}+x_{12}\right) a_{2 j}
$$

Thus $\left[\mathrm{z}-\left(\mathrm{x}_{11}+\mathrm{x}_{12}\right)\right] \mathcal{R}=0$ and so by condition (i), we see that $\mathrm{z}=x_{11}+x_{12}$.
Then $\varphi_{\mathrm{k}}(\mathrm{z})=\varphi_{\mathrm{k}}\left(\mathrm{x}_{11}\right)+\varphi_{\mathrm{k}}\left(\mathrm{x}_{12}\right)$ for all $\mathrm{n}$.

The only essentially different choice for $\mathrm{i}, \mathrm{j}, \mathrm{m}$ is to let $\mathrm{i}=\mathrm{m}=1$ and let $\mathrm{j}=2$.In this case we are led to $\mathcal{R}\left[\mathrm{z}-\left(x_{11}+x_{12}\right)\right]=0$ and so once again $\mathrm{z}=x_{11}+x_{12}$ in view of condition (ii).

Lemma 2.2: $-\varphi_{k}$ is additive map on $\mathcal{R}_{12}$

Proof :-

Let $x_{12}, u_{12} \in \mathcal{R}_{12}$ and $\mathrm{z} \in \mathcal{R}$ such that

$$
\varphi_{k}(z)=\varphi_{k}\left(x_{12}\right)+\varphi_{k}\left(u_{12}\right)
$$

An arbitrary $\mathrm{a}_{1 \mathrm{j}} \in \mathcal{R}_{1 \mathrm{j}}$, we have

$$
\begin{aligned}
\varphi_{\mathrm{k}}\left(\mathrm{za}_{1 \mathrm{j}}\right) & =\sum_{\mathrm{i}=1}^{\mathrm{k}} \varphi_{\mathrm{i}}(\mathrm{z}) \varphi_{\mathrm{i}}\left(\mathrm{a}_{1 \mathrm{j}}\right) \\
& =\sum_{i=1}^{k}\left(\varphi_{i}\left(x_{12}\right)+\varphi_{i}\left(u_{12}\right)\right) \varphi_{i}\left(a_{1 j}\right) \\
& =\sum_{i=1}^{k} \varphi_{i}\left(x_{12}\right) \varphi_{i}\left(a_{1 j}\right) \\
& =\sum_{i=1}^{k} \varphi_{i}\left(\left(x_{12}+u_{12}\right)\left(a_{1 j}\right)\right)=0
\end{aligned}
$$

Then $\mathrm{za}_{1 \mathrm{j}}=0$

For $a_{2 j} \in \mathcal{R}_{2 j}$

$$
\begin{gathered}
\emptyset_{\mathrm{k}}\left(\mathrm{z} a_{2 j}\right)=\sum_{\mathrm{i}=1}^{\mathrm{k}} \varphi_{\mathrm{i}}(\mathrm{z}) \varphi_{\mathrm{i}}\left(\mathrm{a}_{2 j}\right) \\
=\sum_{\mathrm{i}=1}^{\mathrm{k}}\left(\varphi_{\mathrm{i}}\left(\mathrm{x}_{12}\right)\right. \\
\left.+\varphi_{\mathrm{i}}\left(\mathrm{u}_{12}\right)\right) \varphi_{\mathrm{i}}\left(\mathrm{a}_{2 \mathrm{j}}\right) \\
=\sum_{\mathrm{i}=1}^{\mathrm{k}}\left(\varphi_{\mathrm{i}}\left(\mathrm{e}_{1}\right)+\varphi_{\mathrm{i}}\left(\mathrm{x}_{12}\right)\right)\left(\varphi_{\mathrm{i}}\left(\mathrm{a}_{2 \mathrm{j}}\right)\right. \\
\left.+\varphi_{\mathrm{i}}\left(\mathrm{u}_{12}\right)\right) \varphi_{\mathrm{i}}\left(\mathrm{a}_{2 \mathrm{j}}\right) \\
=\sum_{\mathrm{i}=1}^{\mathrm{k}}\left(\varphi_{\mathrm{i}}\left(\mathrm{e}_{1}\right)+\varphi_{\mathrm{i}}\left(\mathrm{x}_{12}\right)\right)\left(\varphi_{\mathrm{i}}\left(\mathrm{a}_{2 \mathrm{j}}\right)\right. \\
\left.+\varphi_{\mathrm{i}}\left(\mathrm{u}_{12} \mathrm{a}_{2 \mathrm{j}}\right)\right) \\
=\sum_{\mathrm{i}=1}^{\mathrm{k}} \varphi_{\mathrm{i}}\left(\mathrm{e}_{1}+x_{12}\right)\left(\mathrm{a}_{2 \mathrm{j}}+\mathrm{u}_{12} \mathrm{a}_{2 \mathrm{j}}\right)
\end{gathered}
$$




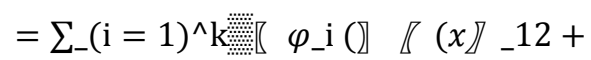
u_12)a_2j)

by using of [Lemma 2.1],

$z \mathrm{a}_{2 \mathrm{j}}=\left(x_{12}+\mathrm{u}_{12}\right) \mathrm{a}_{2 \mathrm{j}}$

$\left[z-\left(x_{12}+\mathrm{u}_{12}\right)\right] \mathrm{a}_{2 \mathrm{j}}=0$ it follows that

$\left[z-\left(x_{12}+\mathrm{u}_{12}\right)\right] \mathcal{R}=0$

And so by condition (i), $z=x_{12}+\mathrm{u}_{12}$

Lemma2.3: $-\varphi_{\mathrm{k}}$ is additive on $\mathcal{R}_{11}$.

Proof :- Let $\mathrm{x}_{11}, \mathrm{u}_{11} \in \mathcal{R}_{11}$, and we write $\varphi_{\mathrm{k}}(\mathrm{z})=\varphi_{\mathrm{k}}\left(\mathrm{x}_{11}\right)+\varphi_{\mathrm{k}}\left(\mathrm{u}_{11}\right)$ for some $\mathrm{z} \in \mathcal{R}$.

Using [Lemma 2.2], we see that

$$
\varphi_{k}\left(z a_{12}\right)=\sum_{i=1}^{k} \varphi_{i}(z) \varphi_{i}\left(a_{12}\right)
$$

$=\sum_{i=1}^{n}\left(\emptyset_{i}\left(x_{11}\right)+\emptyset_{i}\left(u_{11}\right)\right) \emptyset_{i}\left(a_{12}\right)$

$=\sum_{i=1}^{k} \varphi_{i}\left(x_{11}\right) \varphi_{i}\left(a_{12}\right)+\varphi_{i}\left(u_{11}\right) \varphi_{i}\left(a_{12}\right)$

$=\sum_{i=1}^{n} \varphi_{i}\left(x_{11} a_{12}\right)+$

$\varphi_{i}\left(u_{11} a_{12}\right)=\varphi_{k}\left(x_{11} a_{12}\right)+\varphi_{k}\left(u_{11} a_{12}\right)$

$=\varphi_{k}\left(x_{11} a_{12}+u_{11} a_{12}\right)=\varphi_{k}\left(\left(x_{11}+u_{11}\right) a_{12}\right)$

This show that

$z \mathrm{a}_{12}=\left(\mathrm{x}_{11}+\mathrm{u}_{11}\right) \mathrm{a}_{12}$

In other words,

$\left[z-\left(\mathrm{x}_{11}+\mathrm{u}_{11}\right)\right] \mathcal{R}_{12}=0$

Next, we write $z=z_{11}+z_{12}+z_{21}+z_{22}$

And note that

$$
\begin{aligned}
& \begin{aligned}
\varphi_{\mathrm{k}}(\mathrm{z}) & =\varphi_{\mathrm{k}}\left(\mathrm{x}_{11}\right)+\varphi_{\mathrm{k}}\left(\mathrm{u}_{11}\right) \\
& =\varphi_{\mathrm{k}}\left(\mathrm{e}_{1} \mathrm{x}_{11}\right)+\varphi_{\mathrm{k}}\left(\mathrm{e}_{1} \mathrm{u}_{11}\right) \\
& =\sum_{i=1}^{k} \varphi_{\mathrm{i}}\left(\mathrm{e}_{1}\right) \varphi_{\mathrm{i}}\left(\mathrm{x}_{11}\right)+\varphi_{\mathrm{i}}\left(\mathrm{e}_{1}\right) \varphi_{\mathrm{i}}\left(\mathrm{u}_{11}\right)
\end{aligned} \\
& =\sum_{i=1}^{k} \varphi_{\mathrm{i}}\left(\mathrm{e}_{1}\right)\left(\varphi_{\mathrm{i}}\left(\mathrm{x}_{11}\right)+\varphi_{\mathrm{i}}\left(\mathrm{u}_{11}\right)\right) \\
& =\varphi_{\mathrm{k}}\left(\mathrm{e}_{1} z\right)=\varphi_{\mathrm{k}}\left(\mathrm{e}_{1}\left(\mathrm{z}_{11}+\mathrm{z}_{12}+\mathrm{z}_{21}+\mathrm{z}_{22}\right)\right) \\
& =\varphi_{\mathrm{k}}\left(\mathrm{z}_{11}+\mathrm{z}_{12}\right)
\end{aligned}
$$

This equation show that

$\mathrm{Z}=\mathrm{z}_{11}+\mathrm{z}_{12}$, whence $\mathrm{z}_{21}=\mathrm{z}_{22}=0$

By repeating the argument with $e_{1}$ multiplied in to the right, one finds that $\mathrm{z}_{12}=0$,thus yielding $\mathrm{z}=\mathrm{z}_{11} \in \mathcal{R}_{11}$. Therfore $\mathrm{z}-\left(\mathrm{x}_{11}+\mathrm{u}_{11}\right) \in \mathcal{R}_{11}$ and our previous conclusion that $\left(\mathrm{z}-\left(\mathrm{x}_{11}+\mathrm{u}_{11}\right)\right) \mathcal{R}_{12}=0$

Forces $\mathrm{z}=\left(\mathrm{x}_{11}+\mathrm{u}_{11}\right)$ ]from [condition,(iii)].

\section{Lemma2.4:-}

$\varphi_{\mathrm{k}}$ is additive on $\mathrm{e}_{1} \mathcal{R}=\mathcal{R}_{11}+\mathcal{R}_{12}$.

\section{Proof:-}

Let $\mathrm{x}_{11}, \mathrm{u}_{11} \in \mathcal{R}_{11}$ and $\mathrm{x}_{12}$ and $\mathrm{u}_{12} \in \mathcal{R}_{12}$ then by Lemma 2.2,2.3,2.4, we get

$$
\begin{gathered}
\varphi_{\mathrm{k}}\left(\left(\mathrm{x}_{11}+\mathrm{x}_{12}\right)+\left(\mathrm{u}_{11}+\mathrm{u}_{12}\right)\right) \\
=\varphi_{\mathrm{k}}\left(\mathrm{x}_{11}+\mathrm{u}_{11}\right) \\
+\varphi_{\mathrm{k}}\left(\mathrm{x}_{12}+\mathrm{u}_{12}\right) \\
=\varphi_{\mathrm{k}}\left(\mathrm{x}_{11}\right)+\varphi_{\mathrm{k}}\left(\mathrm{u}_{11}\right)+\varphi_{\mathrm{k}}\left(\mathrm{x}_{12}\right)+\varphi_{\mathrm{k}}\left(\mathrm{u}_{12}\right) \\
=\varphi_{\mathrm{k}}\left(\mathrm{x}_{11}+\mathrm{x}_{12}\right)+\varphi_{\mathrm{k}}\left(\mathrm{u}_{11}+\mathrm{u}_{12}\right)
\end{gathered}
$$

Then $\varphi_{\mathrm{k}}$ is additive on $\mathrm{e}_{1} \mathcal{R}=\mathcal{R}_{11}+\mathcal{R}_{12}$

\section{Theorem 2.5:-}

Let $\mathcal{R}$ be a ring containing $\left\{e_{\alpha}: \alpha \in \Lambda\right\}$ family of idempotents which satisfies:

(i) $\mathrm{x}=\{0\} \Rightarrow \mathrm{x}=0$;

(ii)If $e_{\alpha} \mathcal{R} \mathrm{x}=\{0\} \forall \alpha \in \Lambda$, then $\mathrm{x}=0$ (and hence $\mathcal{R} \mathrm{X}=\{0\} \Rightarrow \mathrm{X}=0$.

(iii) $\forall \alpha \in \Lambda, e_{\alpha} \mathrm{x} e_{\alpha} \mathcal{R}\left(1-e_{\alpha}\right)=\{0\} \Rightarrow e_{\alpha} x e_{\alpha}=$ 0 .

Then any multiplicative higher isomorphism $\varphi$ of $\mathcal{R}$ on to an arbitrary ring $\mathrm{S}$ is additive .

\section{Proof}

Let $\mathrm{x}, \mathrm{y} \in \mathcal{R}$, and write

$$
\varphi_{\mathrm{k}}(\mathrm{z})=\varphi_{\mathrm{k}}(\mathrm{x})+\varphi_{\mathrm{k}}(\mathrm{y})
$$

For $\alpha \in A$,select any $t_{\alpha} \in e_{\alpha} \mathcal{R}$.Then

$$
\begin{aligned}
& \varphi_{\mathrm{k}}\left(t_{\alpha} \mathrm{z}\right)=\sum_{\mathrm{i}=1}^{\mathrm{k}} \varphi_{\mathrm{i}}\left(t_{\alpha}\right) \varphi_{\mathrm{i}}(\mathrm{z}) \\
& =\sum_{\mathrm{i}=1}^{\mathrm{k}} \varphi_{\mathrm{i}}\left(t_{\alpha}\right)\left(\varphi_{\mathrm{i}}(\mathrm{x})+\varphi_{\mathrm{i}}(\mathrm{y})\right) \\
& =\sum_{\mathrm{i}=1}^{\mathrm{k}}\left(\varphi_{\mathrm{i}}\left(t_{\alpha}\right) \varphi_{\mathrm{i}}(\mathrm{x})+\varphi_{\mathrm{i}}\left(t_{\alpha}\right) \varphi_{\mathrm{i}}(\mathrm{y})\right) \\
& =\varphi_{\mathrm{k}}\left(t_{\alpha} \mathrm{x}+t_{\alpha} y\right)
\end{aligned}
$$

Since $\varphi_{\mathrm{k}}$ is additive on $e_{\alpha} \mathcal{R}$.by [Lemma 2.4]

Hence $t_{\alpha} z=t_{\alpha}(x+y)$ 
And so we have proved that

$e_{\alpha} \mathcal{R}[z-(x+y)]=0$ for all $\alpha \in \mathrm{A}$.

By [Condition, (ii)] $\Rightarrow \mathrm{z}=\mathrm{x}+\mathrm{y}$. This say that

$$
\varphi_{\mathrm{k}}(x+y)=\varphi_{\mathrm{k}}(\mathrm{x})+\varphi_{\mathrm{k}}(\mathrm{y}) .
$$

\section{Corollary 2.6:-}

Let $\mathcal{R}$ be a prime ring containing idempotent $\mathrm{e} \neq 0,1(\mathcal{R}$ needn't have identity), then any higher multiplicative isomorphism of $\mathcal{R}$ onto a ring $S$ is additive .

\section{Corollary 2.7:-}

Let $\mathcal{R}$ satisfies the conditions of the theorem (or the preceding corollary ).Then any higher multiplicative anti-isomorphism $\varphi$ of $\mathcal{R}$ onto an arbitrary ring $\mathrm{S}$ is additive .

Proof:-Let $\tau$ be the higher anti-isomorphism of $\mathrm{S}$ onto the opposite ring $S^{*}$ of $\mathrm{S}$

By the theorem $\sigma=\tau \varphi$ is an additive of $\mathcal{R}$ onto $S^{*}$ and so $\varphi$ is additive .

\section{References}

[1] R.E. Johnson, Rings with unique addition ,Proc.Amer.Math.Soc. 9 (1958) 57 61.

[2]W.S.Martindal III,When are Multiplicative mapping Additive ?,Proceeding of the American mathematical Society ,21,695698,1969 .

[3] C.E. Rickart, one- to- one mappings of rings and lattices ,Bull .Amer . Math .

Soc.54(1948),758-764.

[4] R.C. Shaheen, on Additive Mappings of Matrix Rings, Phd .Thesis, Baghdad University,(2016).

[5]Y.Wang, The additivity of Multiplicative Maps on Rings,Communication in algebra, Vol 37,(2009),2351-2356.

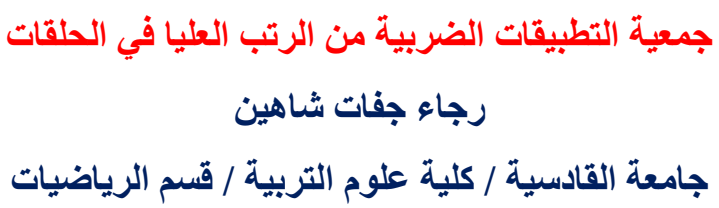

